\title{
Study on the Metal Grating Optical Coupling for Quantum Cascade Detector
}

\author{
L. Li, D.Y. Xiong* \\ Key Laboratory of Polar Materials and Devices, Ministry of Education, East China Normal University, \\ Shanghai 200241, People's Republic of China
}

\begin{abstract}
We present the simulation results of a method for the quantum cascade detectors (QCD) optical coupling. A metal grating was integrated with a QCD to convert normal-incident electromagnetic (EM) waves into non-normal, higher-order propagating wave (HOPW) and surface plasmon wave (SPW), which contribute to excite the bound-to-bound (BTB) intersubband transition of carriers in the QCD. The presented results have significance for the QCD optical coupling design and optimization.
\end{abstract}

\section{INTRODUCTION}

There has been considerable interest in electronic phenomena in semiconductor quantum structure device over past two decades ${ }^{[1-5]}$. Based on bound-to-bound (BTB) intersubband transitions in a built-in asymmetric conduction band structure, quantum cascade detectors (QCD) have the advantage of a photovoltaic scheme and narrow detection line-width [1-2]. Thus this kind of photodetectors has no dark current and low background noise. This leads to substantial potential applications, such as infrared remote sensing, early warning and large-area focal plane arrays. However, due to the polarization selection rule of intersubband transitions, normal incident radiation absorption is forbidden when the radiation is perpendicular to the quantum well layers ${ }^{[3-5]}$. As so far, most work on QCD has been directed to employing a $45^{\circ}$ polished edge geometry to couple infrared radiation into the device. However, for most technical application, planar device geometry and large area normal illumination is quite essential ${ }^{[6-7]}$. In this work, the reflective properties of metal grating for the QCD optical coupling have been studied by the finite-difference time-domain (FDTD) methods. A new choice for metal grating design has been presented due to the exciting of surface plasmon waves (SPW). The presented results may have significance for the QCD optical coupling design and optimization.

\section{RESULTS AND DISCUSSIONS}

To simulate the higher-order propagating wave (HOPW),
SPW and electric field component distribution we have used finite-difference time-domain methods ${ }^{[8]}$. The parameters of the QCD were selected to achieve a peak absorption wavelength around $9 \mu \mathrm{m}$ with a BTB transition.

The simulated optical coupling structure has been shown in Fig. 1, where the source is a normally incident plane wave. After optimizing the electric field intensity distribution around the wavelength of $9.3 \mu \mathrm{m}$, where our QCD device works, we selected the period $p$ of the metal grating as $3.0 \mu \mathrm{m}$, the diameter $d$ as $2.9 \mu \mathrm{m}$, and the thickness of grating layer $h$ as $45 \mathrm{~nm}$.

Figure 2 presents the simulated spectrum of $E_{\mathrm{z}}$ intensity in the $x-y$ plane at the center of the active region at the peak absorption wavelength of QCD. It has been simulated that the electric field enhancement ratio by the SPW is more than six. The intensity of $\left|E_{\mathrm{z}}\right|$ just represents the amount of light which can be absorbed by the QCD.

Figure 3 shows $\left|E_{\mathrm{z}}\right|$ distribution in the $x-y$ plane $(\mathrm{z}=0 \mu \mathrm{m})$ at one center of the grating hole region at the peak absorption wavelength of QCD. The circles in the figure represent the corresponding metal grating hole perforated in the top Au film. Strong HOPWs and SPWs are stimulated in the plane around the hole and the electric field component $E_{\mathrm{z}}$ is enhanced because of the SPW. Figure 4 illustrates $\left|E_{\mathrm{z}}\right|$ distribution in the $x-z$ plane starting from the metal grating/QCD interface at the $y$ point with the peak $\left|E_{\mathrm{z}}\right|$. A large $E_{\mathrm{z}}$ component almost covers the entire device in the active region and the $E_{\mathrm{z}}$ intensity is still very strong even at $1.0 \mu \mathrm{m}$ below the metal grating/QCD interface $(z=-1.0 \mu \mathrm{m})$. Therefore, most of the QCD region can effectively receive and absorb the EM waves with a considerable $E_{\mathrm{z}}$ component. Figure 5 shows $E_{\mathrm{z}}$ intensity

*Correspondence Email: dyxiong@ee.ecnu.edu.cn 


\section{NUSOD 2013}

spectrum vs the normal incident wavelength, which is just around the peak detection wavelength of the QCD.

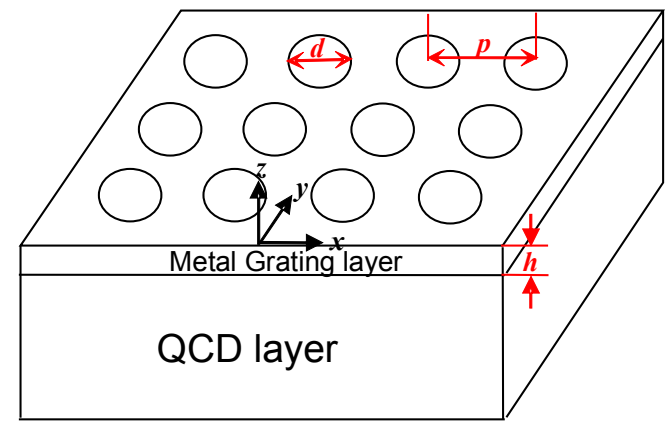

FIG. 1. The schematic of the simulated structure

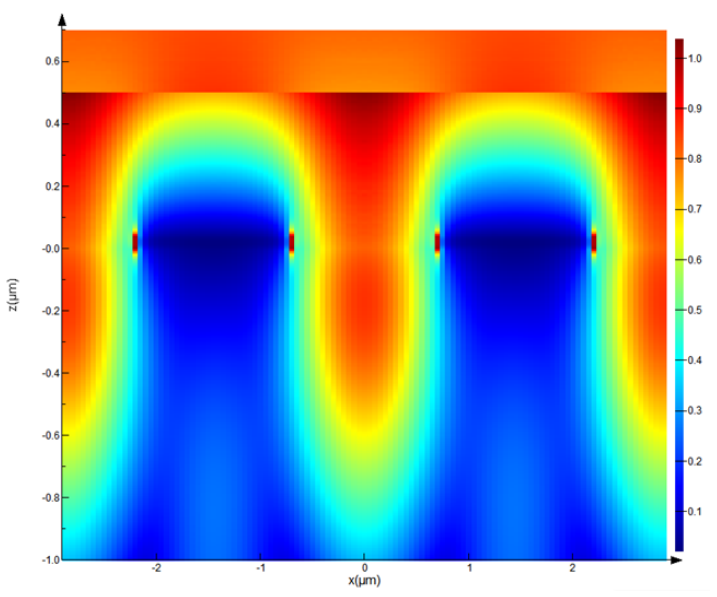

FIG. 2. $E_{\mathrm{z}}$ intensity in the $x-y$ plane at the center of the active region at the peak absorption wavelength of QCD

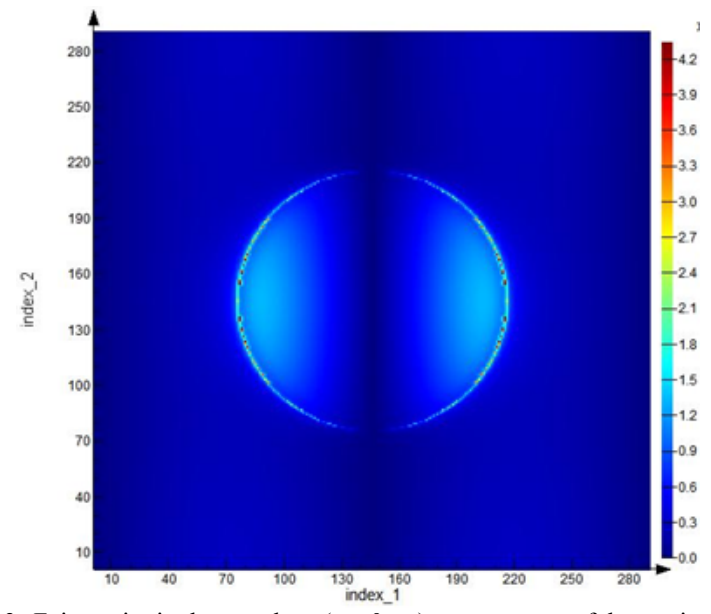

FIG. 3. $E_{\mathrm{z}}$ intensity in the $x-y$ plane $(\mathrm{z}=0 \mu \mathrm{m})$ at one center of the grating hole region at the peak absorption wavelength of QCD

To sum up, we demonstrated strongly coupled HOPWs and SPWs with intersubband electron transitions in a QCD device. The coupled QCD works at normal light incidence. We believe this method may provide an attractive way for fabrication of high performance QCD.

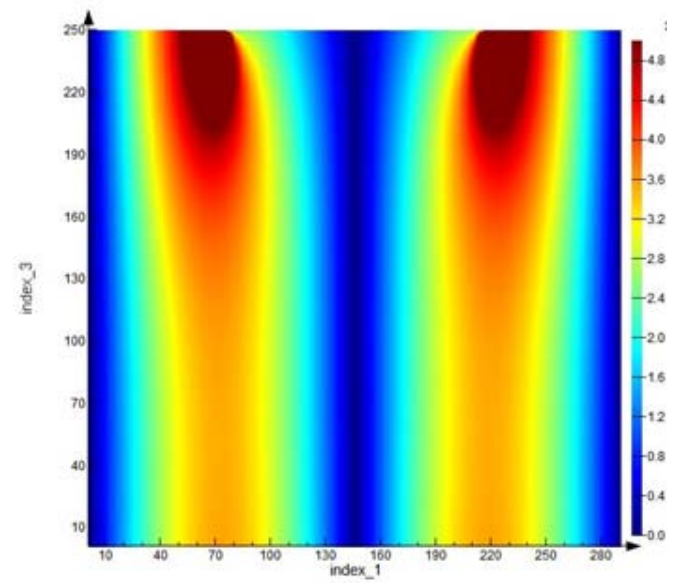

FIG. 4. $E_{\mathrm{z}}$ intensity in the $x-y$ plane $(\mathrm{z}=-1 \mu \mathrm{m})$ at one center of the grating hole region at the peak absorption wavelength of QCD

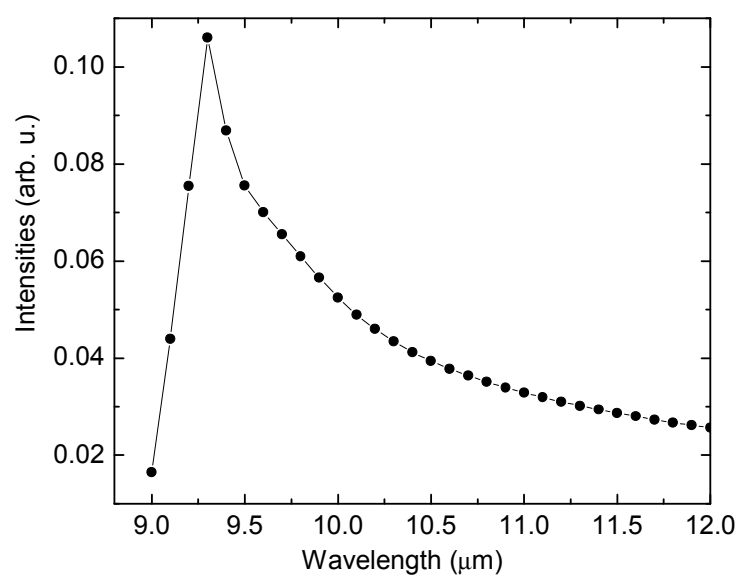

FIG. 5. $E_{\mathrm{z}}$ intensity spectrum vs the normal incident wavelength

\section{ACKOWLEDGEMENTS:}

This work was partially supported by the National Nature Science Foundation of China (Grant No. 61106092).

\section{REFERENCE}

[1] F.R. Giorgetta, E.Baumann, M.Graf, et.al., IEEE J. Quantum Electron. 45, 1039 (2009)

[2] S.Q. Zhai, J.Q. Liu, F.Q. Liu, and Z.G. Wang, Appl. Phys. Lett, 100, 181104 (2012)

[3] S.C. Shen, Microelectronics Journal, 25, 713 (1994)

[4] W. Lu, Y.L. Ji, G.B. Chen, N.Y. Tang, et.al., Appl. Phys. Lett., 83, $4300(2003)$

[5] W. Lu, Y. Fu, Encyclopedia of Nanoscience and Nanotechnology, 9, 179 (2004)

[6] W. Lu, H.J. Ou, M.H. Chen, et.al., Int. J. Infrared. Millim. Waves, 15, 137 (1994)

[7] X.Q. Liu, N. Li, X.S. Chen, W. Lu, W.L. Xu, X.Z. Yuan, et.al. Jpn. J. Appl. Phys. 38, 5044 (1999)

[8] A. Taflove, S. C. Hagness, Computational Electrodynamics:

The Finite-Difference Time-Domain Method (Artech House2000). 